Abstract Wide-angle, polarization-independent structural reflective colors from both directions based on a one-dimensional photonic crystal are demonstrated. Our device produces a distinct and saturated color with high angular tolerant performance up to $\pm 70^{\circ}$ for any polarization state of an incident light wave, which is highly desirable for a broad range of research areas. Moreover, the purity of the color and luminous intensity of the proposed device are improved as compared to conventional colorant-based color filters and colloidal glasses. The present approach may have the potential to replace existing color filters and pigments and pave the way for various applications, including color displays and image sensor technologies.
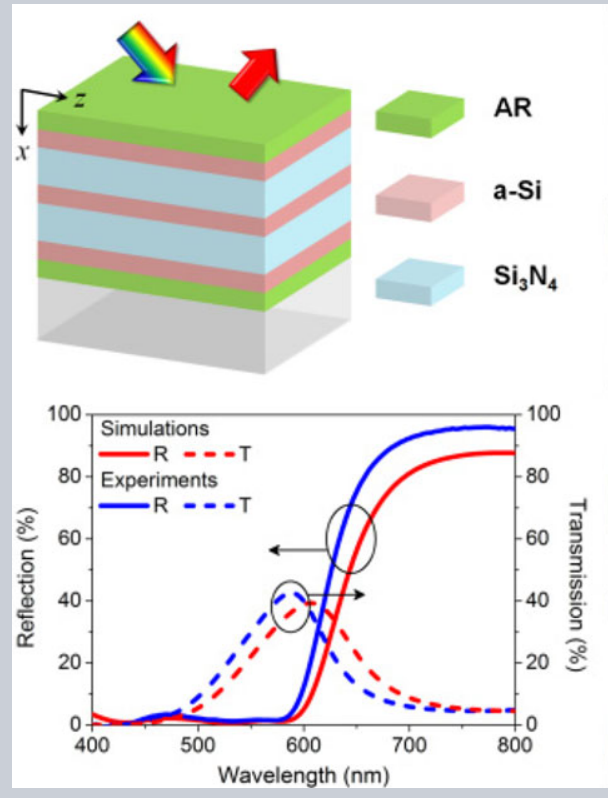

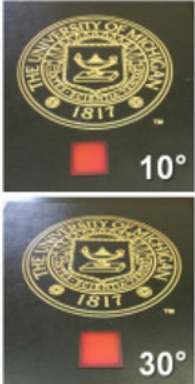

$30^{\circ}$

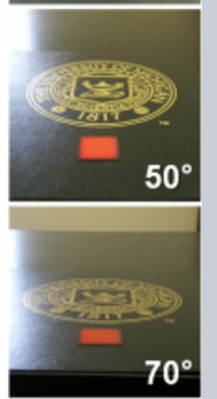

\title{
Angular- and polarization-independent structural colors based on 1D photonic crystals
}

\author{
Kyu-Tae Lee ${ }^{1}$, Chengang $\mathrm{Ji}^{1}$, Debasish Banerjee ${ }^{2, *}$, and L. Jay Guo ${ }^{1, *}$
}

\section{Introduction}

In recent years, renewed attention has been drawn to structural colors for their great promise in a wide variety of fields such as optical measurement systems, light modulators, light-emissive diodes, imaging sensors, colored display devices, and high-chroma pigments [1-5]. These structural colors have significant advantages over the traditional colorant-based, typically an organic dye or chemical pigment, color filtering technologies in terms of high efficiency, slim dimension, long-term stability, being free from photobleaching, but most importantly for their unique metallic and iridescent appearance.

Over the last decade, many research groups have proposed various plasmonic nanostructures, including nanorods, nanowires, and nanocavities [6-15]. In addition to the plasmonic structures, numerous subwavelength grating-based photonic designs have been utilized to achieve the filtering capability in the visible wavelength range [16-21]. However, a suppression of an angular dependence remained challenging. This is due to the fact that a light-coupling scheme, for example a prism and grating coupling, is required to couple the incident light wave to the surface plasmon or guided mode resonant modes.
Although great efforts are being made to generate the structural colors with the angle-independent characteristic, the scalability of these proposed designs still remains questionable [3-5, 22, 23]. Besides, most of the previously reported studies utilize the one-dimensional (1D) grating structure that works for only one polarization $[8,18,23]$. Furthermore, the most widely used geometries are metal-dielectric-metal (MDM) designs that involve an optically thick bottom metal layer, thus resulting in a color only from the illumination direction [24-28]. Unless such bottlenecks are addressed, it is extremely difficult to use these designs as an alternative to the conventional color filters.

Herein, we present a new scheme that provides a distinct color that is insensitive to wide range of viewing angles and irrespective of the polarization states of incident light. By adopting a 1D photonic crystal (PC) configuration, the proposed device shows angular robust reflective color up to $\pm 70^{\circ}$ for both transverse electric (TE) and transverse magnetic (TM) polarized light. In addition, a symmetric structure design enables the same reflection color from both the top and bottom sides, which allows these films to be used as pigment flakes in paint [29]. Furthermore, the spectral profile of our structural color has almost negligible offresonance wavelengths, leading to great color purity with

\footnotetext{
${ }^{1}$ Department of Electrical Engineering and Computer Science, The, University of Michigan, Ann Arbor, Michigan, 48109, USA

${ }^{2}$ Toyota Research Institute of North America, Ann Arbor, Michigan, 48105-9748, USA

*Corresponding author: e-mail: debasish.banerjee@ tema.toyota.com; guo @umich.edu
} 
high luminous intensity, which has not been observed by using a colloidal nanoparticle array approach. The strategy described in this study may open up a new path to the extensive use of the structural colors for various applications that are difficult with conventional techniques.

\section{Experimental materials and methods}

\subsection{Device fabrication}

A glass substrate is piranha cleaned (mixture of $\mathrm{H}_{2} \mathrm{O}_{2}$ and $\mathrm{H}_{2} \mathrm{SO}_{4}$ with $1: 1$ volume ratio) for $30 \mathrm{~min}$. Silicon nitride $\left(\mathrm{Si}_{3} \mathrm{~N}_{4}\right)$ and amorphous silicon (a-Si) materials are deposited by employing plasma-enhanced chemical vapor deposition (PECVD) at a rate of 1.3 and $1 \AA^{-1}\left(\mathrm{SiH}_{4}\right.$ gas with 13.56 MHz RF power), respectively.

\subsection{Simulations}

Transfer matrix method-based simulations are performed to calculate the spectral reflectance curves at normal incidence, angle-resolved reflection spectra, optical field distributions, and optimized refractive index for the antireflection (AR) coating. Wavelength-dependent refractive indices of $\mathrm{Si}_{3} \mathrm{~N}_{4}$ and a-Si are measured with a spectroscopic ellipsometer (M-2000, J. A. Woollam) and utilized in the simulation.

\subsection{Optical measurements}

The measurement of the spectral reflectance curve of the fabricated sample at normal incident angle is conducted by using a thin-film measurement instrument (F20, Filmetrics) integrated with a spectrometer and light source. The angle-resolved reflection spectra for both TE and TM polarizations are measured by using a spectroscopic ellipsometer (M-2000, J. A. Woollam).

\section{Results and discussions}

A schematic diagram of a proposed wide-angle, polarization-independent reflection structural color is shown in Fig. 1a, where the structure is composed of alternating layers of $\mathrm{Si}_{3} \mathrm{~N}_{4}$ and a-Si. Even though our strategy can be used to produce various colors, we are targeting a high-color-purity angle-insensitive red color that has proved difficult to achieve [30]. In the case of the structural colors, creating a resonance at longer wavelengths (i.e., red color) requires a thicker layer, thus causing a sensitivity with respect to the angle of incidence due to the longer thickness as compared to other colors. Such an angle-sensitive attribute could be addressed by employing high-index materials. However, it requires a large number of periodic units with a low optical contrast [31]. In

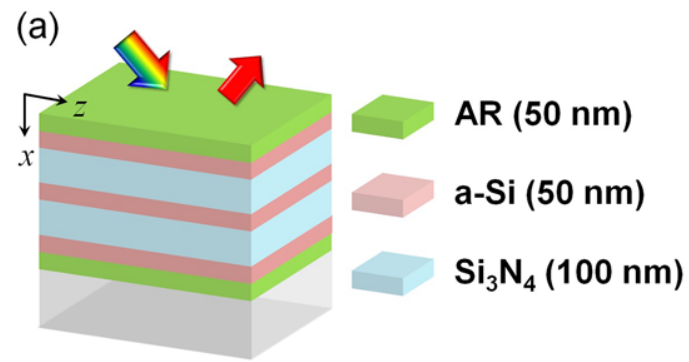

(b)
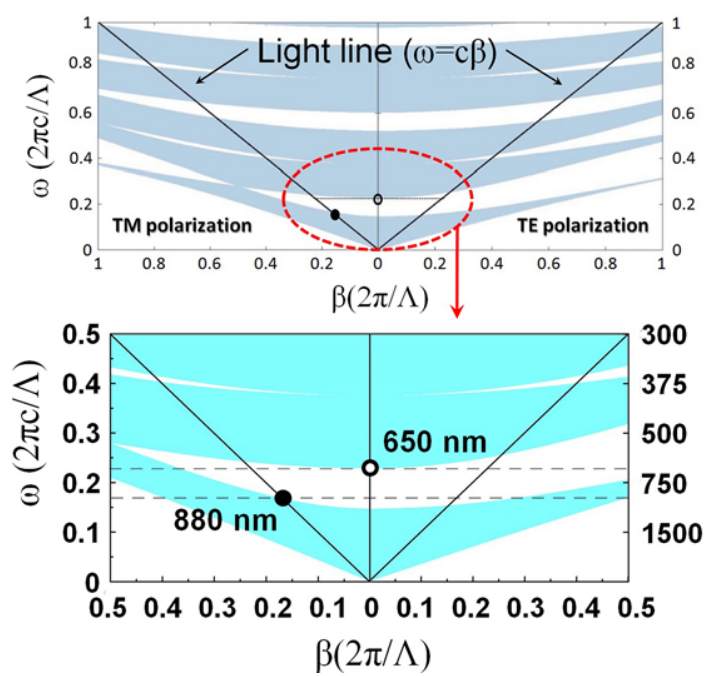

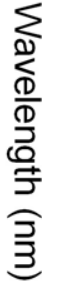

Figure 1 (a) A schematic diagram of a proposed polarizationindependent structural color with wide viewing angles based on a 1D photonic crystal (PC). (b) A projected band structure for our proposed multilayer system without the antireflection (AR) layers, which is periodic in the $\mathrm{x}$ direction with $n_{1}=2$ and $n_{2}=4$. The electromagnetic modes for TM and TE polarizations are described to the left and right of the origin. An enlarged view of the red dotted region is shown below.

addition, a higher-order resonance appears at a shorterwavelength range, which degrades the color purity. We design our PC structure to have the band edge located around $600 \mathrm{~nm}$ in order to reflect strong red colors. The center wavelength $\left(\lambda_{c}\right)$ of a photonic stopband (i.e., high reflection window) is designed to be $800 \mathrm{~nm}$. The thickness of each layer required to achieve strong constructive interferences of the reflected light at $\lambda_{\mathrm{c}}$ is a quarter wavelength $\left(d_{1,2}=\frac{\lambda_{c}}{4 n_{1,2}}\right)$, which leads to 100 and $50 \mathrm{~nm}$ of thickness for $\mathrm{Si}_{3} \mathrm{~N}_{4}\left(n_{1}=2\right)$ and a-Si $\left(n_{2}=4\right)$ layer, respectively. The bandwidth $\left(\Delta \lambda_{\mathrm{c}}=\frac{4 \lambda_{\mathrm{c}}}{\pi} \sin ^{-1}\left(\frac{n_{2}-n_{1}}{n_{2}+n_{1}}\right)\right)$ of the photonic stopband of our designed structure is $350 \mathrm{~nm}$, so that it generates strong reflection at wavelengths beyond $\sim 600 \mathrm{~nm}$. Figure $1 b$ depicts a band structure for the quarter-wave stack proposed in this paper with $n_{1}=2\left(\mathrm{Si}_{3} \mathrm{~N}_{4}\right.$ at $\lambda_{\mathrm{c}}=$ $800 \mathrm{~nm})$ and $n_{2}=4\left(\mathrm{a}-\mathrm{Si}\right.$ at $\left.\lambda_{\mathrm{c}}=800 \mathrm{~nm}\right)$. Generally, for a $1 \mathrm{D}$ periodically stratified medium with a refractive index that is periodic in the $x$ direction and a wave propagating in the $x-z$ plane, the wave vectors $k_{x}$ in $x$ and $\beta$ in $z$ represent the electromagnetic modes. The projected band 
(a)

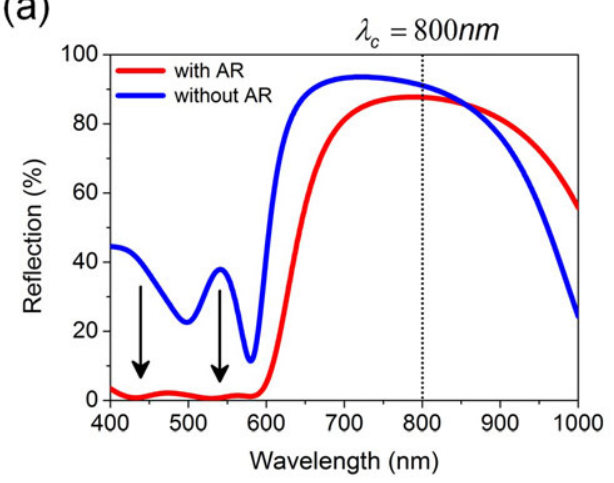

(b)
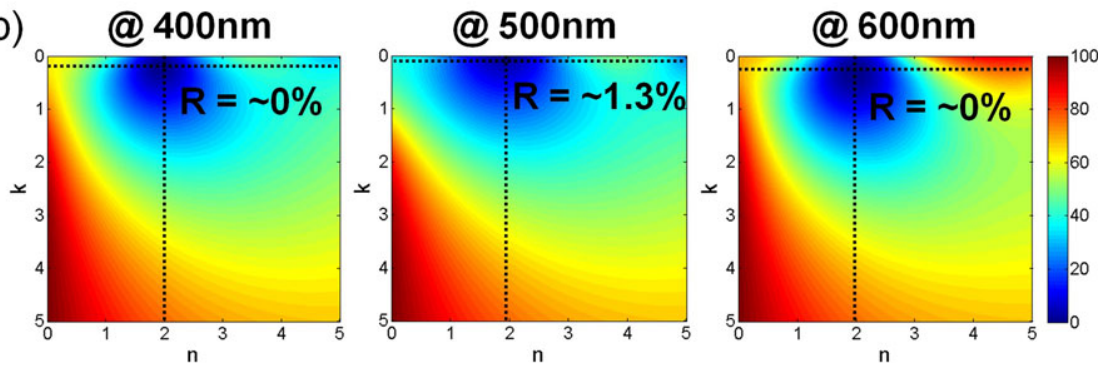

Figure 2 (a) Calculated spectral reflectance curves with (red solid line) and without (blue solid line) an AR coating showing that the reflections from $400 \mathrm{~nm}$ to $600 \mathrm{~nm}$ are greatly suppressed by adding the AR layer. (b) Calculated contour plots of the reflection as a function of a complex index of refraction (real $(n)$ and imaginary $(k)$ parts) of the 50-nm thick AR layer at 400, 500, and $600 \mathrm{~nm}$, respectively. It is found that nearly insignificant reflections smaller than $\sim 1.3 \%$ occur in the wavelength regimes between $400 \mathrm{~nm}$ and $600 \mathrm{~nm}$ when $n$ and $k$ are equivalent to 1.98 and 0.14 , respectively. Color maps denote the intensity values of the reflected light. structure is obtained by utilizing the dispersion relationship $K(\beta, \omega)=(1 / \Lambda) \cos ^{-1}[1 / 2(A+D)]$ for the Bloch wave function based on the Floquet theorem, where $K$ is the Bloch wave number, $\omega$ is the mode frequency and $A$ and $D$ are the transfer matrix elements for the periodic structure [32]. There exist electromagnetic modes in the shaded regions, while the white regions represent the photonic stopband. Moreover, the electromagnetic modes in the surrounding medium obey $\omega=c \sqrt{k_{x}^{2}+\beta^{2}}$, where $c$ is the speed of light in air and $k_{x}$ is the wave vector in the $x$ direction, and those modes can be represented by the whole area above $\omega>c \beta$, as shown in the figure. In Fig. 1b, the forbidden band for TM modes shrinks to zero when $\beta=(\omega / c) n_{2} \sin \theta_{\mathrm{B}}$ with the Brewster's angle $\theta_{\mathrm{B}}=\tan ^{-1}\left(n_{1} / n_{2}\right)$, where the Fresnel reflection on the multilayer interfaces vanishes. The criterion for omnidirectional reflection behavior is that no incident light can be coupled into the structure from air, which means there exists a frequency zone where the band structure of the stack does not overlap with $\omega>c \beta$. In the visible wavelength, this frequency range is found to be $0.17<\omega \Lambda / 2 \pi c<0.23$, indicated as the filled and open circles in Fig. 1b, and the corresponding wavelength range is between $650 \mathrm{~nm}$ and $880 \mathrm{~nm}$. Our proposed structure consists of a high refractive index (i.e., a-Si) material so that a refracted angle into the structure can be very small by Snell's law $\left(n_{1} \sin \theta_{1}=n_{2} \sin \theta_{2}\right)$, thus being able to achieve the angle-insensitive performance. We calculate a slope $\left(\frac{d \omega}{d \beta}\right)$ of the band diagram for both the proposed device and the multilayer structure comprising an air and $\mathrm{Si}_{3} \mathrm{~N}_{4}$ (i.e., low refractive index materials). The low refractive index material-based multilayer system shows a steeper slope for both TE and TM polarizations than the structure that is composed of the high refractive index, which is given in Supporting Information. As can also be seen from an enlarged view of Fig. 1b, a relative flat band structure with respect to $\beta$ can be achieved due to the high refractive index of the a-Si material, which will be experimentally validated in the last section of the paper.

Based on this design the reflections from $400 \mathrm{~nm}$ to $600 \mathrm{~nm}$ are $\sim 33 \%$ due to a high index contrast between air and a-Si as shown in Fig. 2a (blue solid line). To address this problem, we add an AR layer, and simulation is carried out to mitigate the reflections in this wavelength range. Figure $2 \mathrm{~b}$ shows the calculated reflections as functions of real $(n)$ and imaginary $(k)$ parts of the refractive index of the AR layer with a given thickness $(50 \mathrm{~nm})$ at 400 , 500 , and $600 \mathrm{~nm}$. It is apparent that the reflection value is almost negligible (less than $\sim 1.3 \%$ ) in the wavelength regions from $400 \mathrm{~nm}$ to $600 \mathrm{~nm}$ when $n$ and $k$ are equal to 1.98 and 0.14 , respectively. We note that the decreased (or increased) thickness of the AR layer could be used with the increased (or decreased) $n$ value of the $\mathrm{AR}$ film. Experimentally, we choose $\mathrm{Si}_{3} \mathrm{~N}_{4}$ as the AR coating material because its refractive index is fairly close to the optimized index needed for the AR effect (i.e., $n=2$ ). Note that other dielectric materials with the index close to 2, such as $\mathrm{WO}_{3}, \mathrm{MoO}_{3}$, and $\mathrm{Ta}_{2} \mathrm{O}_{5}$, can also be used for the AR layer. We also investigate the AR coating by utilizing an optical admittance diagram to further validate its effect [33-35]. The optical admittance $(Y=\sqrt{\varepsilon / \mu})$ is the inverse of the impedance and widely used at optical frequencies to describe the characteristic of the thin-film multilayer structure due to negligible magnetic effects, which lead to its numeric value being equal to the complex refractive index of a material, although their physical meanings are 


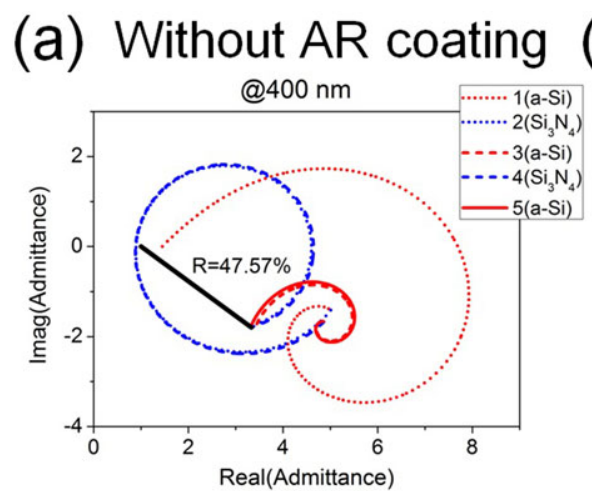

\section{(b) With AR coating}
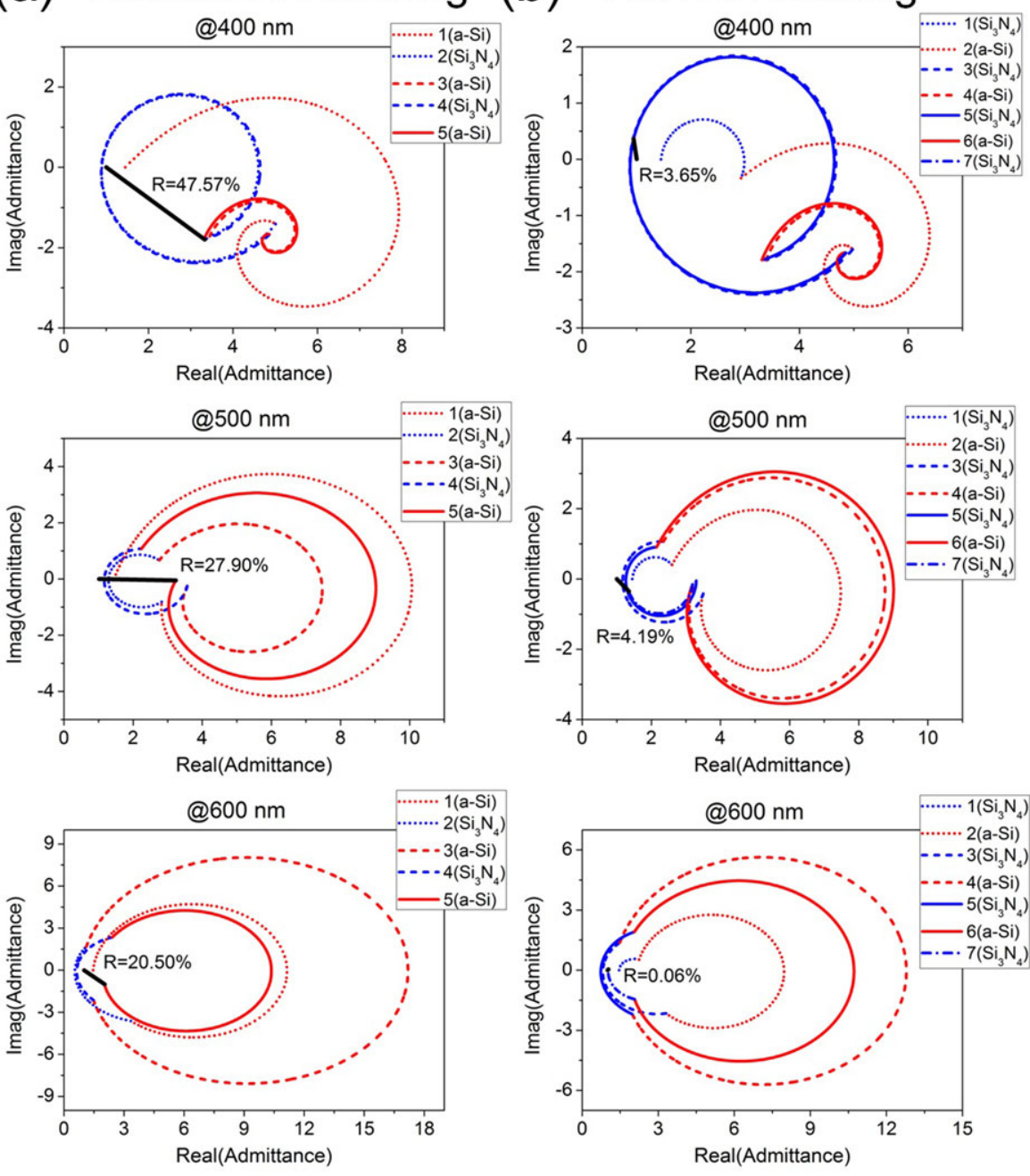

Figure 3 Optical admittance diagrams of the proposed PC-based multilayer structure (a) without and (b) with AR coating layers $(50 \mathrm{~nm}$ $\mathrm{Si}_{3} \mathrm{~N}_{4}$ ) at 400,500 , and $600 \mathrm{~nm}$. An initial admittance point is the glass substrate and the following locus is determined by the thickness and refractive index of the medium. It is clear that the distance from the final admittance point to the air is considerably decreased after adding the AR layers, achieving greatly reduced reflections in a short-wavelength range from $400 \mathrm{~nm}$ to $600 \mathrm{~nm}$ and thus excellent color purity. different. At normal incidence, the amplitude of the reflection coefficient of a surface is expressed by the following equation:

$$
r=\frac{Y_{0}-Y}{Y_{0}+Y},
$$

where $Y_{0}$ is the incident medium's optical admittance and $Y$ is the optical admittance of the surface, respectively. After substituting $r=|r| e^{i \phi}=\alpha+$ $i \beta$, where $\phi=\frac{2 \pi n}{2} d \cos \left(\theta_{\text {inc }}\right), d=$ thickness, and $\theta_{\text {inc }}=$ incidence angle and $Y=x+i y$ into Eq. (1), then

$$
|r| e^{i \phi}=\frac{Y_{0}-x-i y}{Y_{0}+x+i y} .
$$

Multiplying each side of Eq. (2) and its complex conjugate together leads to

$$
x^{2}+y^{2}-2 Y_{0}\left(\frac{1+r^{2}}{1-r^{2}}\right) x+Y_{0}^{2}=0 .
$$

Equation (3) describes the optical admittance of the surface and the resulting admittance is the equation of a circle on the real axis in the case of transparent dielectric media or perfect electric conductors. In absorbing medium cases such as semiconductors or real metals, however, the extinction coefficient of the material renders the admittance locus somewhat distorted, thus yielding spirals [36]. The optical admittance of the structure begins with the point of the substrate $\left(n_{\text {sub }}, 0\right)$. As the thickness of the dielectric medium is increased, the admittance rotates on the circle. A zero reflection is achieved by perfectly matching the admittance of the system with that of the air (i.e., the admittance terminates at the air $(1,0)$ ). This also suggests that the reflection can be suppressed depending on how close the air and the last admittance points are. In Figs. 3a and b, the optical admittance diagrams of the proposed PC-based structural colors without and with the AR coating at 400, 500 , and $600 \mathrm{~nm}$ are presented. Paths of the optical admittance traced out by the a-Si and $\mathrm{Si}_{3} \mathrm{~N}_{4}$ layers are represented by a red and blue colored line, respectively. The black solid 
(a)

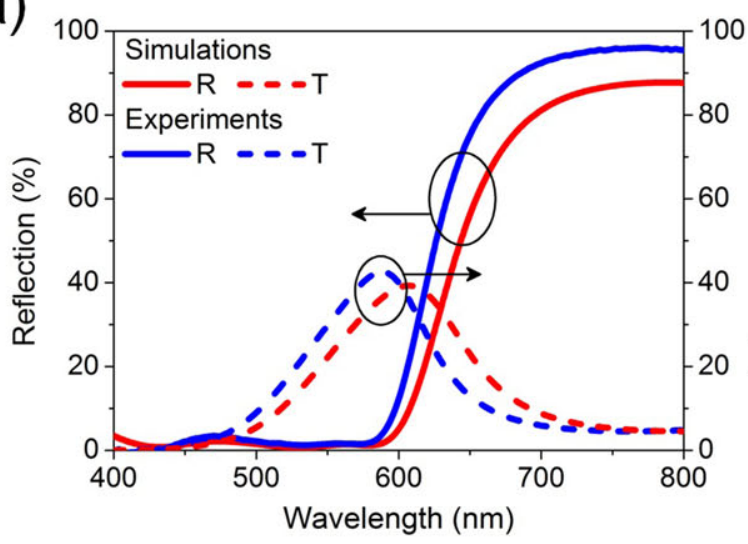

(c)

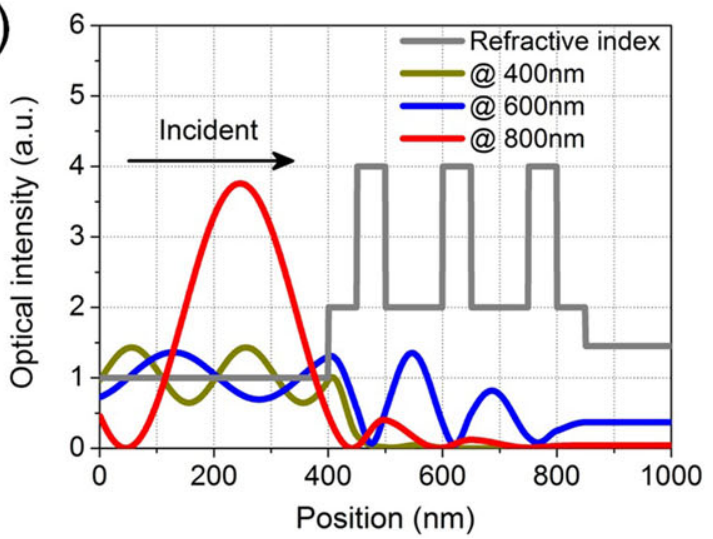

(b)
Simulation

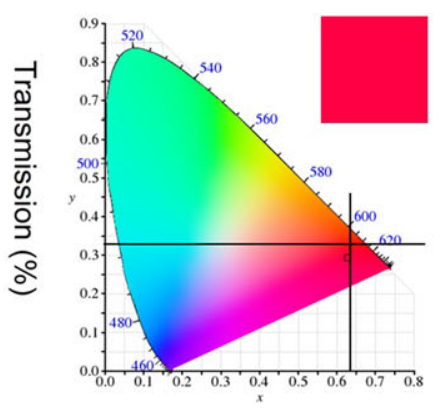

Experiment

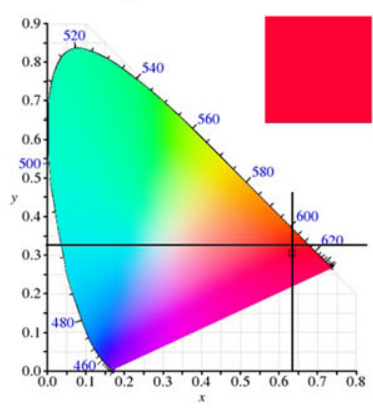

(d)

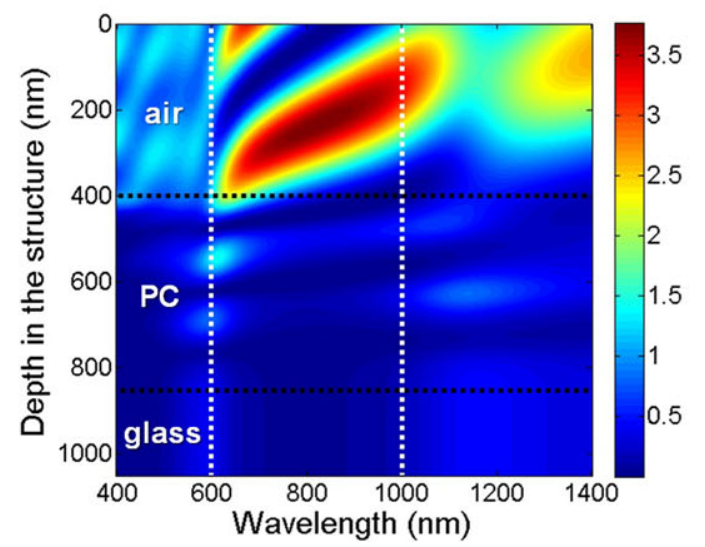

Figure 4 (a) Calculated and measured spectral reflectance (solid lines) and transmittance (dotted lines) curves at normal incidence, both of which are in good agreement. (b) An illustration of the color coordinate from both the simulation and experiment depicted on the CIE 1931 chromaticity diagram. The crossing point of the two black solid lines signifies a standard red color coordinate $(0.64,0.33)$ for the liquid crystal display devices. (c) Optical intensity distributions of the electric field at 400 (dark yellow), 600 (blue), and $800 \mathrm{~nm}$ (red) together with the refractive-index profile (gray), incident from the left-hand side. The interference of the counterpropagating light waves leads to the oscillating intensity outside the PC structure (i.e., air from 0 to $400 \mathrm{~nm}$ in position). The strong absorption of the a-Si medium at $400 \mathrm{~nm}$ results in little field penetration, while the light of $800 \mathrm{~nm}$ can get fully reflected from the PC structure. (d) A field penetration into our designed PC multilayer structure as a function of wavelength. The color map denotes the optical intensity inside the structure.

line indicates the distance from the termination point to the air, along with the expected reflection that can be computed by the following equation $\left(R=\left(\frac{Y_{0}-Y_{1}}{Y_{0}+Y_{1}}\right)\left(\frac{Y_{0}-Y_{1}}{Y_{0}+Y_{1}}\right)^{*}\right)$, where $Y_{0}$ and $Y_{1}$ are the optical admittance of the starting incident medium and ending point). Calculated coordinates of the final admittance obtained without the AR coating are $(4.85,-1.66),(3.24,-0.05)$, and $(2.03,-1.02)$ for 400 , 500 , and $600 \mathrm{~nm}$ wavelengths, respectively. These admittance points are fairly far from that of the air, which gives rise to a strong reflection at that wavelength, as can be seen from the calculated reflection values. On the contrary, the distance between the air and the termination point is highly reduced by adding the AR layer and the corresponding coordinates are $(0.95,0.38),(1.35,-0.34)$, and $(1.03,0.04)$, all of which exhibit significantly suppressed reflections of less than $5 \%$. We study the influence of the thickness of the AR coating medium on the reflection, which is found in the Supporting Information. Both $\mathrm{Si}_{3} \mathrm{~N}_{4}$ and a-Si materials are deposited by PECVD. The refractive indices of deposited $\mathrm{Si}_{3} \mathrm{~N}_{4}$ and a-Si are measured by a spectroscopic ellipsometer (M-2000, J. A. Woollam Co.) and given in the Supporting Information. After applying the AR coating to our design, the resulting spectral response shows significantly suppressed reflections between 400 and $600 \mathrm{~nm}$, as represented by the red solid line in the Fig. 2a, which will be confirmed by the experiment in the following section.

In Fig. 4a, the calculated and measured reflection (solid curves) and transmission (dotted curves) spectra at normal incidence angle are depicted. A transfer matrix method based on the measured refractive indices of $\mathrm{Si}_{3} \mathrm{~N}_{4}$ and a-Si is used to plot the calculated spectra and the spectral profiles of the fabricated sample is measured by using a thin-film measurement system (F20, Filmetrics) incorporated with the light source and spectrometer. Excellent agreement is obtained between the simulation and experimental data. We note that the measured spectral responses show blueshifted profiles with relatively higher intensity as compared to the calculated spectra, which 

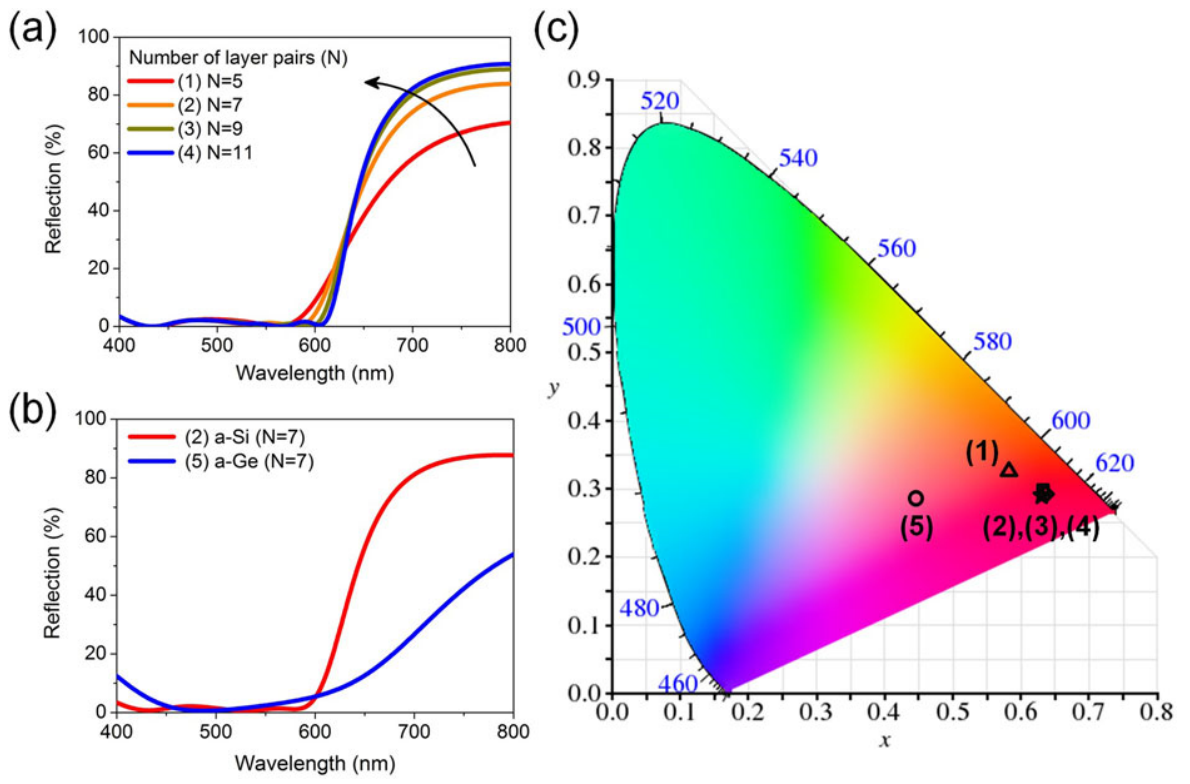

Figure 5 (a) Calculated reflection responses with different numbers of layer pairs from 5 to 11 . (b) Calculated spectral reflectance curves of the PC that is composed of 7-layer stacks made of a-Si/ $\mathrm{Si}_{3} \mathrm{~N}_{4}$ and a-Ge/Si $\mathrm{N}_{4}$ pairs. (c) A representation of the color coordinate from the reflection spectra studied in (a) and (b) described on the CIE 1931 chromaticity diagram. could be attributed to the fact that the refractive indices of both $\mathrm{Si}_{3} \mathrm{~N}_{4}$ and a-Si materials for the fabricated samples are slightly lower than those used in the simulation. It is also clear that the measured reflection spectrum has a sharper slope at $650 \mathrm{~nm}$ than the simulated profile, which is attributed to the lower optical absorption loss (absorption $=$ $\frac{2 \pi c \varepsilon_{0} n k}{\lambda}|E(x, \lambda)|^{2}$, where $c$ : speed of light, $\varepsilon_{0}$ : permittivity of free space, $n$ : real part of the refractive index, $k=\frac{\lambda \alpha}{4 \pi}$ : extinction coefficient, $\alpha$ : absorption coefficient) resulting from the lower refractive indices (i.e., high Q-factor) [24]. In particular, strong reflections from $600 \mathrm{~nm}$ to $800 \mathrm{~nm}$ are achieved, while the reflections at shorter wavelength regions (i.e., 400-600 $\mathrm{nm}$ ) are greatly reduced, thereby creating a red reflective color with high color purity. The luminous intensity of our targeted color $(\sim 96 \%)$ obtained from the experiment is much improved as compared with that of the traditional organic dye-based red color $(\sim 70 \%)$. In order to evaluate the purity of the produced red color, the color coordinates $(x, y)$ are calculated from the calculated and measured reflection spectra, and described on the CIE 1931 chromaticity diagram, as presented in Fig. 4b. It is noted that the crossing point of the two black solid lines indicates a standard red coordinate $(0.64,0.33)$ for the liquid crystal display (LCD) devices. The color coordinates $(x, y)$ for the simulation and experiment are $(0.655,0.294)$ and $(0.656,0.321)$, respectively, both of which are quite close to the standard red point $(0.64,0.33)$, indicating that the red color of our design shows great color purity. We note that the approach described here could be expanded to create blue and green colors as well by reducing the thickness of each medium with appropriate thicknesses of the AR coating layer, while sacrificing the luminous intensity to some extent due to the absorption loss of the semiconductor layer at shorter wavelengths. Optical-intensity distributions of the electric field at different wavelengths are shown in Fig. 4c. The light is incident from the left-hand side where the incident medium is the air (0-400 $\mathrm{nm}$ in position) and passes through the PC structure (400-850 $\mathrm{nm}$ in position) on the glass substrate $(850-1000 \mathrm{~nm}$ in position). The gray solid line represents the profile of refractive indices of air, $\mathrm{Si}_{3} \mathrm{~N}_{4}, \mathrm{a}-\mathrm{Si}$, and $\mathrm{SiO}_{2}$ corresponding to $1,2,4$, and 1.45 , respectively. The field inside the PC at $400 \mathrm{~nm}$ (dark yellow) is almost 0 that is due to the strong absorption of the a-Si layer, whereas the light of $600 \mathrm{~nm}$ (blue) can get through the whole PC structure and thus be partially transmitted. It can also be seen that the field intensity in the incident medium at $800 \mathrm{~nm}$ (i.e., $\lambda_{\mathrm{c}}$ ) is much stronger than that at either 400 or $600 \mathrm{~nm}$, implying that a significant portion of the light for $800 \mathrm{~nm}$ is reflected from the PC. To clearly see how the optical field penetrates into the PC structure for the whole visible-wavelength range, Fig. $4 d$ describes the field distribution as functions of wavelength and depth in the structure. As can be seen from the figure, there is trivial field penetration in the PC but a strong field in the air within the photonic stopband (i.e., 600 and $1000 \mathrm{~nm}$ ).

Next, we examine the influence of the number of pairs and the selection of the semiconductor material on the color purity. Figure 5a depicts the calculated reflection spectra depending on how many layer pairs the PC structure has. It is expected that increasing the number of pairs (i.e., combination of a-Si and $\mathrm{Si}_{3} \mathrm{~N}_{4}$ ) enables a steeper photonic stopband and thus stronger reflection, because more constructive interferences occur with increasing the number of layer pairs. Next, we compare the contribution of different semiconductor layers. In Fig. 5b, the calculated reflection spectra of the PC structure comprising either 7-layer pairs of a-Si/ $/ \mathrm{Si}_{3} \mathrm{~N}_{4}$ (red solid line) or amorphous germanium $(\mathrm{a}-\mathrm{Ge}) / \mathrm{Si}_{3} \mathrm{~N}_{4}$ (blue solid line) are shown. As is seen in the figure, the spectral reflectance of the PC involving a-Si/ $\mathrm{Si}_{3} \mathrm{~N}_{4}$ combination has a much sharper profile than that of the $\mathrm{a}-\mathrm{Ge} / \mathrm{Si}_{3} \mathrm{~N}_{4}$ case. The broad spectrum for the 
(a)

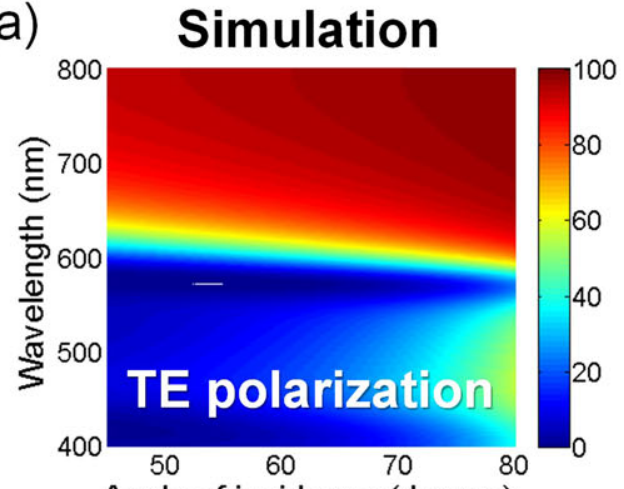

(c)

Angle of incidence (degree)

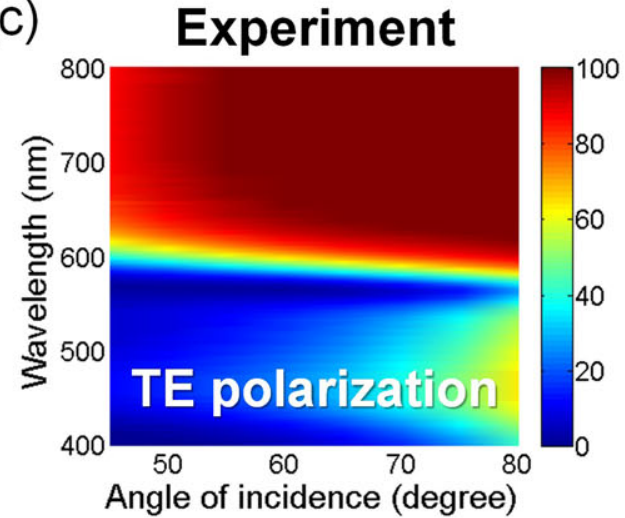

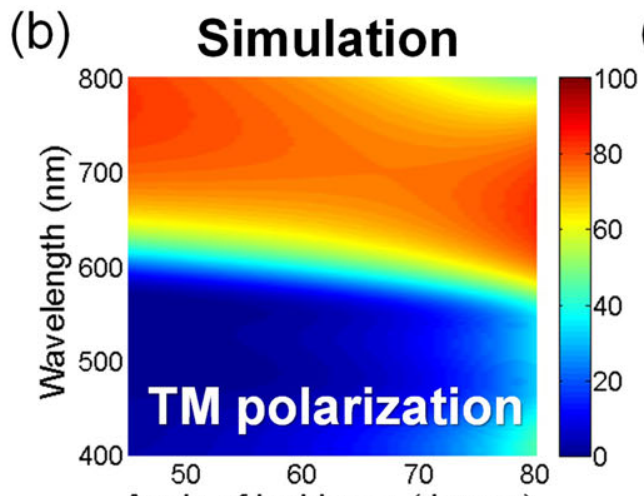

Angle of incidence (degree)

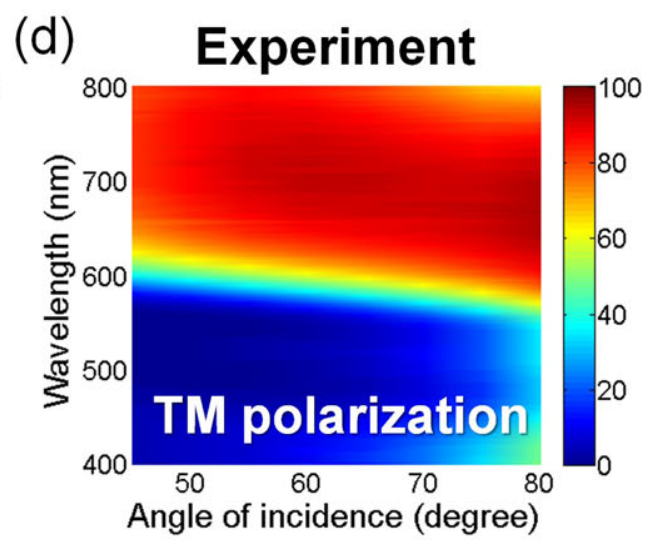

(e)

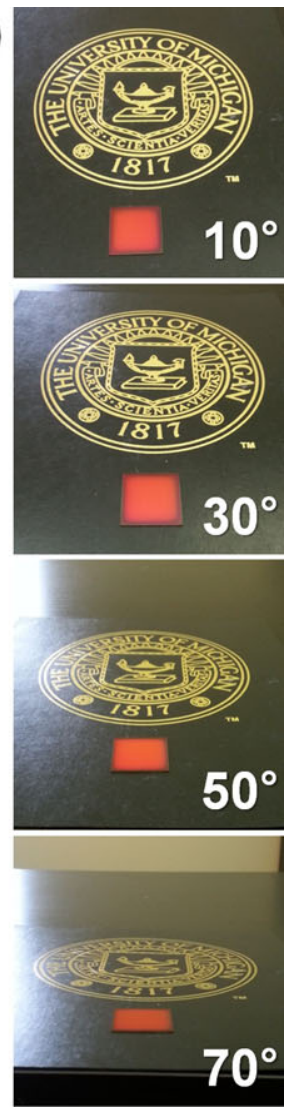

Figure 6 (a) and (b) Calculated angular dependences of the designed device for TE and TM polarizations. (c) and (d) Measured angular dependences corresponding to those in (a) and (b). (e) Optical images of the fabricated device on a black paper at the four oblique angles of incidence are taken with the indoor light, displaying that the red color can be maintained over a wide angular range. Note that the black paper is used to prevent the reflected light from interfering with the transmitted light.

latter case is attributed to a large absorption of the a-Ge material across the entire visible-wavelength spectrum, which results in a poor quality factor (Q-factor) of the PC. To estimate how the color purity varies depending on the number of stacks of layers and the choice of the semiconductor, the color coordinates $(x, y)$ of the reflection spectra discussed in Figs. 5a and b are calculated and represented on the CIE 1931 chromaticity diagram, as shown in Fig. 5c. When the number of layer pairs is 5 , the photonic stopband is not sharp enough to achieve the pure red color, because it contains a small portion of the yellow light, thus the calculated color coordinate lies midway between the yellow and red (i.e., orange color). When the number of stacks is greater than 7, it is evident that the purity of the red color is improved due to the increased number of the constructive interferences. Since the color purity of the PC that contains 7 pairs is fairly comparable to that of the PC consisting of 9 and 11 layer stacks, we choose the PC device with 7-layer pairs as a demonstration. The spectral reflectance of the PC made of 7-layer pairs of a-Ge and $\mathrm{Si}_{3} \mathrm{~N}_{4}$ has some portions of the blue wavelengths, which lead to the color coordinate shifting toward the bluish region (i.e., magenta color).
Lastly, we explore the dependence of our proposed structure on the angle and polarization states of the incident light. The calculated and measured angle-resolved reflection spectra for both TE and TM polarizations are presented in Figs. 6a-d. The simulation data are obtained from a transfer matrix method and the angular behavior of the fabricated device is measured by using a spectroscopic ellipsometer (M-2000, J. A. Woollam Co.) from $45^{\circ}$ to $80^{\circ}$, showing a great match with the simulation. As can be seen from both the simulation and experiment, the strong reflections from $600 \mathrm{~nm}$ to $800 \mathrm{~nm}$ with highly suppressed reflections below $600 \mathrm{~nm}$ are achieved over a large angle of incidence for both polarizations, which was expected from the band structure, as shown in Fig. 1b. Within the frequency zone (white region) in the projected band structure, there will be a total reflection from any incident angle for both polarizations with a finite number of films, which is consistent with both the simulated and measured angle-resolved reflection spectra presented in Figs. 6a-d. The high refractive index of the a-Si material leads to the relatively flat band structure with respect to $\beta$. For the waves below $650 \mathrm{~nm}$, the reflection is much lower because most lights transmit due to the coupling between incident wave and the modes in 
the structure, and then are absorbed by the a-Si layer and partially transmitted. Optical photographs of the fabricated sample on a black paper at several oblique angles of incidence under ambient light illumination (i.e., unpolarized light) are shown in Fig. 6e. It is clear that the reflective red color can be viewed from various angles with the unpolarized light, thus validating the color characteristics of our design is insensitive to the incidence angle and polarization. It should be noted that the sample is placed on the black paper to absorb the transmitted light that could be interfered with the reflection color. We also note that the red color can be observed from the opposite side since the proposed structure is symmetric and not opaque. Such bidirectionality with the angle-invariant and polarization-independent properties is highly desirable for certain applications. Moreover, the structures could be made into pigment form and applied as a coating layer to an object to produce the desired colors.

\section{Conclusions and outlook}

In conclusion, we proposed and experimentally demonstrated a polarization-independent structural color with improved viewing angles employing a 1D PC structure. Our device exhibits a distinct reflective color with high purity and luminance over a wide angular range up to $\pm 70^{\circ}$ regardless of the incident polarization from both directions. The approach discussed here can be potentially useful for diverse applications, including colored displays and image sensors.

\section{Supporting Information}

Additional supporting information may be found in the online version of this article at the publisher's website.

Acknowledgements. The authors would like to thank Toyota Research Institute of North America, and the National Science Foundation (ECCS 1202046) for the support of this work.

Received: 31 January 2015, Revised: 10 April 2015, Accepted: 24 April 2015

\section{Published online:}

Key words: structural colors, photonic crystals, thin films, wavelength-filtering devices.

\section{References}

[1] F.-J. Ko and H.-P. D. Shieh, Appl. Opt. 39, 1159-1163 (2000).

[2] P. B. Catrysse, W. Suh, S. Fan, and M. Peeters, Opt. Lett. 29, 974-976 (2004).

[3] K. Chung, S. Yu, C.-J. Heo, J. W. Shim, S.-M. Yang, M. G. Han, H.-S. Lee, Y. Jin, S. Y. Lee, N. Park, and J. H. Shin, Adv. Mater. 24, 2375-2379 (2012).

[4] K. Chung and J. H. Shin, J. Opt. Soc. Am. A 30, 962-968 (2013).
[5] J.-G. Park, S.-H. Kim, S. Magkiriadou, T. M. Choi, Y.-S. Kim, and V. N. Manoharan, Angew. Chem. Int. Ed. 53, 28992903 (2014).

[6] G. Si, Y. Zhao, J. Lv, M. Lu, F. Wang, H. Liu, N. Xiang, T. J. Huang, A. J. Danner, J. Teng, and Y. J. Liu, Nanoscale 5, 6243-6248 (2013).

[7] K. Seo, M. Wober, P. Steinvurzel, E. Schonbrun, Y. Dan, T. Ellenbogen, and K. B. Crozier, Nano Lett. 11, 1851-1856 (2011).

[8] T. Xu, Y.-K. Wu, X. Luo, and L. J. Guo, Nature Commun. 1, 59 (2010).

[9] S. Yokogawa, S. P. Burgos, and H. A. Atwater, Nano Lett. 12, 4349-4354 (2012).

[10] T. Xu, H. Shi, Y.-K. Wu, A. F. Kaplan, J. G. Ok, and L. J. Guo, Small 7, 3128-3136 (2011).

[11] D. Inoue, A. Miura, T. Nomura, H. Fujikawa, K. Sato, N. Ikeda, D. Tsuya, Y. Sugimoto, and Y. Koide, Appl. Phys. Lett. 98, 093113 (2011).

[12] M. A. Vincenti, M. Grande, D. de Ceglia, T. Stomeo, V. Petruzzelli, M. De Vittorio, M. Scalora, and A. D’Orazio, Appl. Phys. Lett. 100, 201107 (2012).

[13] Y. S. Do, J. H. Park, B. Y. Hwang, S.-M. Lee, B.-K. Ju, and K. C. Choi, Adv. Opt. Mater. 1, 133-138 (2013).

[14] B. Ai, Y. Yu, H. Möhwald, and G. Zhang, Adv. Opt. Mater. 1, 724-731 (2013).

[15] H. J. Park, T. Xu, J. Y. Lee, A. Ledbetter, and L. J. Guo, ACS Nano 5, 7055-7060 (2011).

[16] H.-S. Lee, Y.-T. Yoon, S.-S. Lee, S.-H. Kim, and K.-D. Lee, Opt. Exp. 15, 15457-15463 (2007).

[17] Y.-T. Yoon, H.-S. Lee, S.-S. Lee, S. H. Kim, J.-D. Park, and K.-D. Lee, Opt. Exp. 16, 2374-2380 (2008).

[18] A. F. Kaplan, T. Xu, and L. J. Guo, Appl. Phys. Lett. 99, 143111 (2011).

[19] C.-H. Park, Y.-T. Yoon, and S.-S. Lee, Opt. Exp. 20, 23769 23777 (2012).

[20] C.-H. Park, Y.-T. Yoon, V. R. Shrestha, C.-S. Park, S.S. Lee, and E.-S. Kim, Opt. Exp. 21, 28783-28793 (2013).

[21] M. G. Han, C.-J. Heo, H. Shim, C. G. Shin, S.-J. Lim, J. W. Kim, Y. W. Jin, and S. Lee, Adv. Opt. Mater. 2, 535-541 (2014).

[22] D. Banerjee and M. Zhang, J. Mod. Opt. 57, 1180-1188 (2010).

[23] Y.-K. R. Wu, A. E. Hollowell, C. Zhang, and L. J. Guo, Sci. Rep. 3, 1194 (2013).

[24] K.-T. Lee, S. Seo, J. Y. Lee, and L. J. Guo, Adv. Mater. 26, 6324-6328 (2014).

[25] K. Kumar, H. Duan, R. S. Hegde, S. C. W. Koh, J. N. Wei, and J. K. W. Yang, Nature Nanotech. 7, 557-561 (2012).

[26] A. S. Roberts, A. Pors, O. Albrektsen, and S. I. Bozhevolnyi, Nano Lett. 14, 783-787 (2014).

[27] K.-T. Lee, S. Seo, J. Y. Lee, and L. J. Guo, Light Sci. Appl. 3, e215; doi:10.1038/lsa.2014.96 (2014).

[28] C. Yang, W. Shen, Y. Zhang, H. Peng, X. Zhang, and X. Liu, Opt. Exp. 22, 11384-11391 (2014).

[29] G.B. Smith, A. Gentle, P. D. Swift, A. Earp, and N. Mronga, Sol. Energ. Mater. Sol. Cells 79, 179-197 (2003).

[30] S. Magkiriadou, J.-G. Park, Y.-S. Kim, and V. N. Manoharan, Phys. Rev. E 90, 062302 (2014). 
[31] D. Banerjee and M. Zhang, J. Mod. Opt. 57, 1180-1188 (2010).

[32] P. Yeh, A. Yariv, and C.-S. Hong, J. Opt. Soc. Am. 67, 423438 (1977).

[33] K.-T. Lee, S. Seo, J. Y. Lee, and L. J. Guo, Appl. Phys. Lett. 104, 231112 (2014).
[34] C.-C. Lee, S.-H. Chen, and C.-C. Jaing, Appl. Opt. 35, 56985703 (1996).

[35] H. Kostlin and G. Frank, Thin Solid Films 89, 287-293 (1982).

[36] H. A. Macleod, Thin-Film Optical Filters (CRC Press, Boca Raton, 2010), Chap. 3. 